\title{
A Facile and Sensitive Colorimetric Approach to Confirming the Presence of Polydopamine Thin Films on (Bio)Material Surfaces
}

\author{
Athina Andrea ${ }^{1}$ and Jason P Manse $/ I^{*}$ \\ ${ }^{1}$ Department of Science and Environment, Roskilde University, Universitetsvej 1, Denmark \\ ${ }^{2}$ Department of Applied Sciences, University of the West of England, Frenchay Campus, Coldharbour Lane, UK
}

\begin{abstract}
Mussel foot adhesive proteins are one of nature's remarkable triumphs; they guarantee a secure and robust bond between the mollusc's byssal threads and the surface to which they settle ensuring that they are securely fastened. We now know that some of these adhesive proteins are especially rich in lysine and 3,4-dihydroxyL-phenylalanine (DOPA) residues. Interestingly dopamine, under mildly alkaline conditions, as would be expected for a marine environment, results in a spontaneous polymerisation reaction, the product of which, polydopamine, tenaciously bonds to a whole raft of different materials, indeed, even polytetrafluoroethylene (PTFE, Teflon) isn't immune from adulteration! Polydopamine in turn is rich in reactive quinone, amino and catechol moieties making it amenable to further modification. Over the past decade researchers have used polydopamine thin films as capturing platforms for the fabrication of functional materials by grafting, for example, biological agents such as enzymes and growth factors to generate bioactive supports. Typically, corroboration of polydopamine bonding to a (bio)material surface requires the use of some sophisticated and costly approaches such as Raman spectroscopy, X-ray reflectivity, atomic force microscopy and contact angle analyses. Herein we provide a simple yet sensitive means to support polydopamine thin film deposition upon a solid surface by utilising an assay procedure more commonly associated with protein determinations, namely that based on bicinchoninic acid. For those wishing to explore the potential for polydopamine to form reactive platforms on whatever surface, our simple assay procedure provides a pragmatic and cheap solution before moving to those more specialised and technically challenging approaches.
\end{abstract}

Keywords

Polydopamine, Biomaterial functionalisation, Chromogenic detection, Bicinchoninic acid

\section{Introduction}

Inspired by the tenacity of marine mussels (Mytilus edulis) to attach to wet surfaces, polydopamine (PDA) thin films represent highly versatile reactive platforms for the facile fabrication of novel (bio)materials [1-3]. Indeed, PDA coatings are currently regarded as "start-of-the-art" functional supports with wide ranging applications [4] Simply steeping materials in mildly alkaline solutions of dopamine hydrochloride (DHC) results in the spontaneous polymerisation to PDA, a brown-black complex analogous to the formation of eumelanin [5]. The tenacious binding of PDA to multiple substrates lends itself for further modification by exploiting the plentiful amino, quinone and catechol moieties [1]. Schiff-base and Michael addition reactions, for example, afford peptide and protein conjugation of PDA coatings in generating a wide spectrum of functional surfaces. Evidence for the successful binding of PDA to the material surface invariably requires the use of technically demanding and costly tools, including Raman spectroscopy, X-ray reflectivity,

\footnotetext{
*Corresponding author: Jason P Mansell, Department of Applied Sciences, University of the West of England, Frenchay Campus, Coldharbour Lane, Bristol, BS16 1QY, UK, E-mail: jason.mansell@uwe.ac.uk
}

Received: April 25, 2018; Accepted: July 02, 2018;

Published online: July 04, 2018

Citation: Andrea A, Mansell JP (2018) A Facile and Sensitive Colorimetric Approach to Confirming the Presence of Polydopamine Thin Films on (Bio)Material Surfaces. Regen Med Ther 2(1):30-36

Copyright: (c) 2018 Andrea A, et al. This is an open-access article distributed under the terms of the Creative Commons Attribution License, which permits unrestricted use, distribution, and reproduction in any medium, provided the original author and source are credited. 
Citation: Andrea A, Mansell JP (2018) A Facile and Sensitive Colorimetric Approach to Confirming the Presence of Polydopamine Thin Films on (Bio)Material Surfaces. Regen Med Ther 2(1):30-36

XPS and atomic force microscopy. In making the first steps towards the development of a new PDA-based technology researchers could benefit from a quick and inexpensive assay to confirm successful PDA conjugation to the test substrate(s). Whilst the exact nature of deposited PDA films is still open for debate it likely comprises a heterogeneous blend of eumelanin-like oligo indoles and catecholamine/quinone/indole heteropolymers [2]. The multiple amino groups associated with PDA are predicted to readily react with a bicinchoninic acid (BCA)based protein assay reagent. Hence a simple and reliable means of confirming the presence of PDA films on test substrates should be easily afforded during the development of novel functional (bio)materials. Herein we report on the successful detection of PDA thin films at different surfaces using a simple BCA-based colorimetric assay. Exposing PDA-coated materials to the assay reagent generates an attractive violet colour as $\mathrm{Cu}^{2+}$ ions are reduced to $\mathrm{Cu}^{+}$. We suggest that the use of such a test could be of significant value in screening different substrates for PDA coatings before commissioning studies employing costlier and technically demanding approaches. In addition, our approach could be particularly beneficial for the purposes of PDA-coating optimisation during the development, for example, of novel implantable bone biomaterials for orthopaedics and dentistry.

\section{Materials and Methods}

\section{Laboratory reagents}

Unless stated otherwise all general reagents were purchased from Sigma (Poole, Dorset, UK). Grit-blasted and nitric acid-passivated medical-grade titanium (Ti6AL4V) discs (10 mm diameter, depth $1.5 \mathrm{~mm}$ ) were supplied by OsteoCare Implant System (Slough, UK). Multiwell (24-well) cell culture plates (Corning (Costar $\left.^{\mathrm{ru}}\right)$, Appleton Woods, Birmingham, UK) served as a source of tissue culture plastic (TCP) for PDA-functionalisation. The BCA-based assay kit (Pierce) was from Fisher Scientific UK Ltd. (Loughborough, UK) and the detecting reagent mixture prepared exactly as instructed on the day of use.

\section{PDA-coating of (bio)materials}

Unless stated otherwise dopamine hydrochloride (DHC) was reconstituted to a final concentration of 2 $\mathrm{mg} / \mathrm{ml}$ in either cell culture grade water, $10 \mathrm{mM}$ Tris ( $\mathrm{pH} 8.5$ ), $10 \mathrm{mM}$ sodium bicarbonate $(\mathrm{pH} 8.3$ ) or $10 \mathrm{mM}$ HEPES ( $\mathrm{pH}$ 7.4). Resultant solutions were applied to either Ti or TCP surfaces within 2-3 minutes of preparation and left exposed for a maximum of 24 hours. Following the desired incubation time, the DHC/PDA solutions were removed, and the surfaces rinsed three times with distilled water prior to PDA coating detection using the
BCA-based assay reagent. For TCP the wells were rinsed using water from a squeezy bottle whereas Ti discs were transferred to universal tubes filled with water $(\sim 25 \mathrm{ml})$ and the samples vigorously shaken to dislodge weakly bound PDA. An assessment of PDA coating extent did not proceed if the final rinse reacted positively with the BCA reagent.

\section{Detection and quantification of PDA thin films using a BCA-based assay reagent}

Evidence of a PDA coating was reliably achieved by treating control and functionalised surfaces with the BCA assay reagent. Once rinsed the TCP wells were treated with $250 \mu \mathrm{l}$ of distilled water followed by $125 \mu \mathrm{l}$ of the freshly prepared BCA reagent (as instructed by the manufacturer) and the resultant solutions left to incubate for 90 minutes at room temperature. Sample aliquots $(0.1$ $\mathrm{ml}$ ) were subsequently transferred to a 96-well plate and readings taken at $540 \mathrm{~nm}$ using a multiwell plate reader (FLUOstar OPTIMA, BMG labtech) operated from a PC using MARS software. For Ti discs, rinsed specimens were placed in clean wells of 24-well tissue culture plates and treated with the BCA reagent exactly as per control and modified TCP. A series of DHC concentrations (0$25 \mathrm{micrograms} / \mathrm{ml}$ ) in distilled water enabled extrapolation of the PDA coating, as DHC equivalents, for the different treated surfaces.

\section{Statistical analysis}

Unless stated otherwise all analyses were made using two-tailed, unpaired, t-tests, assuming unequal variance, for additional statistical rigour. Data between groups was deemed to be statistically significant where $\mathrm{p}<0.05$.

\section{Results}

\section{Polydopamine (PDA) readily reacts with a bicin- choninic acid (BCA)-based assay reagent}

Multiwell tissue culture plastic (TCP) plates were exposed to a $2 \mathrm{mg} / \mathrm{ml}$ solution of DHC in $10 \mathrm{mM}$ sodium carbonate ( $\mathrm{pH} 8.3$ ) for different times (15 mins - $3 \mathrm{hrs)}$ against buffer alone. With increasing time, the incubating solution adopted a darker brown colour confirming the presence of polymeric material. Once incubated, treated and control wells were rinsed and processed for PDA detection using the BCA-based assay reagent. The development of an attractive violet complex consequent to $\mathrm{Cu}^{2+}$ reduction provided clear evidence of PDA presence for each of the different sample wells (Figure 1A). It is evident from the figure presented that increasing the incubation time led to clear increases in violet colour intensity. Similarly, when Ti discs were steeped in the same DHC solution the extent of the PDA coating increased with incubation time (Figure 1B). 
Citation: Andrea A, Mansell JP (2018) A Facile and Sensitive Colorimetric Approach to Confirming the Presence of Polydopamine Thin Films on (Bio)Material Surfaces. Regen Med Ther 2(1):30-36

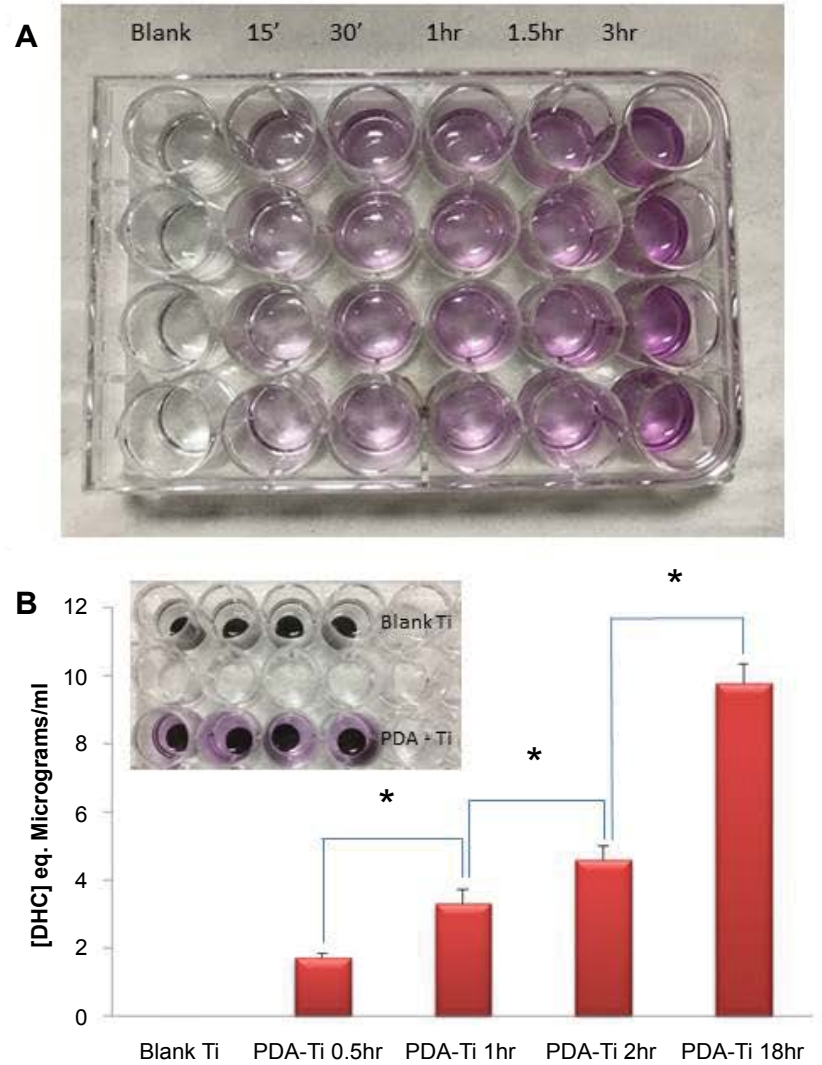

Figure 1: Chromogenic detection of polydopamine (PDA) films using a bicinchoninic acid (BCA) assay reagent.

A) Wells of a tissue culture plastic (TCP) plate were exposed to either buffer alone (blank - $10 \mathrm{mM}$ sodium bicarbonate ( $\mathrm{pH}$ 8.3)) or a $2 \mathrm{mg} / \mathrm{ml}$ solution of dopamine hydrochloride (DHC, $1 \mathrm{ml} / \mathrm{well}$ ) and left to incubate, at room temperature, for a maximum of three hours. At the indicated times solutions were aspirated and the wells rinsed three times with distilled water prior to BCA reagent addition. The development of a violet complex, consequent to $\mathrm{Cu}^{2+}$ reduction, is evidence for PDA at the test surface. Qualitatively, the image depicted (a representative from three independent experiments) provides evidence of PDA formation within 15 minutes with clear increases in colour intensity with incubation time. B) Medical grade titanium discs were either exposed to buffer alone (blank Ti) or a $2 \mathrm{mg} / \mathrm{ml}$ solution of $\mathrm{DHC}$ and the metal samples allowed to incubate for a maximum of 18 hours. At the indicated times Ti discs were recovered, transferred to $30 \mathrm{ml}$ universal tubes and given three vigorous rinses in distilled water with manual shaking. Washed specimens were transferred to clean wells and exposed to the BCA reagent. After a 90 -minute incubation aliquots $(0.1 \mathrm{ml})$ of each sample were transferred to a 96-well plate and the absorbance at $540 \mathrm{~nm}$ taken against a standard series of DHC to extrapolate PDA at the Ti surfaces. Quantitatively, increasing the DHC incubation time results in clear and significant incremental increases ( $" p<0.01)$ in PDA deposition at the Ti surface. All data (a representative from two independent experiments) are expressed as the mean equivalent of $\mathrm{DHC}$ (micrograms $/ \mathrm{ml}$ ) plus the standard deviation from 4 replicates. Inset an image of blank and 18-hour DHC-treated Ti discs following treatment with the BCA reagent.

The extent of TCP modification by PDA is influenced by buffer choice

Multiwell TCP plates were exposed to a $2 \mathrm{mg} / \mathrm{ml}$ solution of DHC using three different buffers; $10 \mathrm{mM}$ sodium bicarbonate ( $\mathrm{pH} 8.3$ ), $10 \mathrm{mM}$ HEPES ( $\mathrm{pH} 7.4$ ) and $10 \mathrm{mM}$ Tris ( $\mathrm{pH} 8.5)$. Treated wells were left for a maximum of 20 hours prior to processing for PDA detection and quantification using the BCA-based assay reagent. As anticipated TCP wells treated with DHC in alkaline buffers yielded the greatest extent of PDA modification $(\mathrm{p}<0.01)$ compared to the neutral buffered HEPES solution (Figure 2). The extent of PDA-functionalisation of TCP between the carbonate and Tris buffers were comparable. When DHC was reconstituted in distilled water there was no evidence of PDA formation upon TCP, indeed there was no clear change in sample appearance over several days indicating the lack/paucity of a polymerisation reaction (data not shown).

\section{Evidence of a clear time and dose-response effect for PDA-functionalisation of TCP}

Multiwell tissue culture plastic (TCP) plates were exposed to varying concentrations of DHC $(0.2-2.0 \mathrm{mg} / \mathrm{ml})$ in $10 \mathrm{mM}$ sodium bicarbonate buffer $(\mathrm{pH} 8.3)$ and the presence of PDA coatings determined at multiple time points. The findings presented (Figure 3) provide clear evidence for a time and dose-related functionalisation event with the highest concentration of DHC $(2.0 \mathrm{mg} / \mathrm{ml})$ for the longest incubation time $(24 \mathrm{hr})$ generating surfaces with the greater extent of PDA-functionalisation. 
Citation: Andrea A, Mansell JP (2018) A Facile and Sensitive Colorimetric Approach to Confirming the Presence of Polydopamine Thin Films on (Bio)Material Surfaces. Regen Med Ther 2(1):30-36

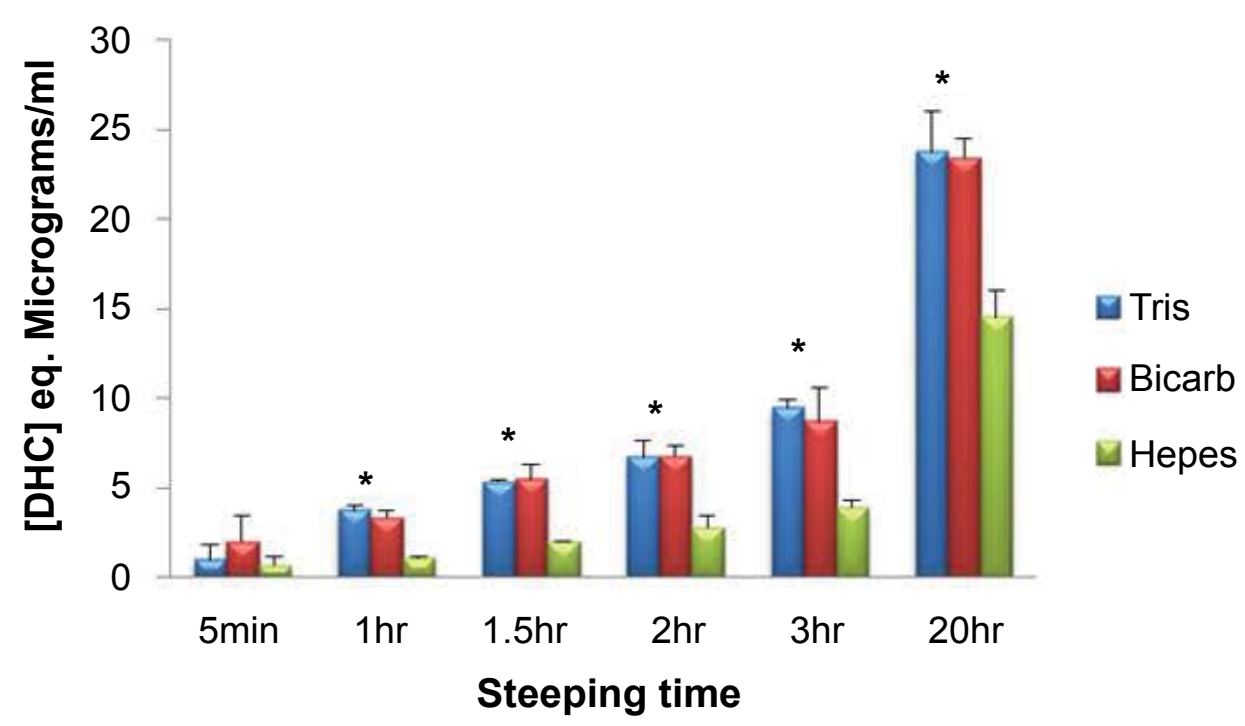

Figure 2: The influence of $\mathrm{pH}$ on the extent of polydopamine (PDA) formation at a tissue culture plastic (TCP) surface. Dopamine hydrochloride (DHC) was reconstituted at $2 \mathrm{mg} / \mathrm{ml}$ in either $10 \mathrm{mM}$ Tris $(\mathrm{pH} 8.5), 10 \mathrm{mM}$ sodium bicarbonate $(\mathrm{pH}$ 8.3) or $10 \mathrm{mM}$ HEPES ( $\mathrm{pH} 7.4)$. Resultant solutions were dispensed into wells of TCP pates (1 ml/well) and left for a maximum of 20 hours. At the desired times the solutions were aspirated, and the wells rinsed three times with distilled water prior to treatment with a bicinchoninic acid (BCA) assay reagent. After a 90-minute room temperature incubation aliquots (0.1 ml) of each sample were transferred to a 96 -well plate and the absorbance at $540 \mathrm{~nm}$ taken against a standard series of DHC to extrapolate PDA at the TCP surfaces. From the data depicted both the Tris and bicarbonate buffers support a similar extent of PDA formation. However, compared to these buffers, the neutral HEPES solution yielded surfaces with less PDA for each of the incubation times ( $(p<0.01)$. All data (a representative from two independent experiments) are expressed as the mean equivalent of $\mathrm{DHC}$ (micrograms $/ \mathrm{ml}$ ) plus the standard deviation from 4 replicates.

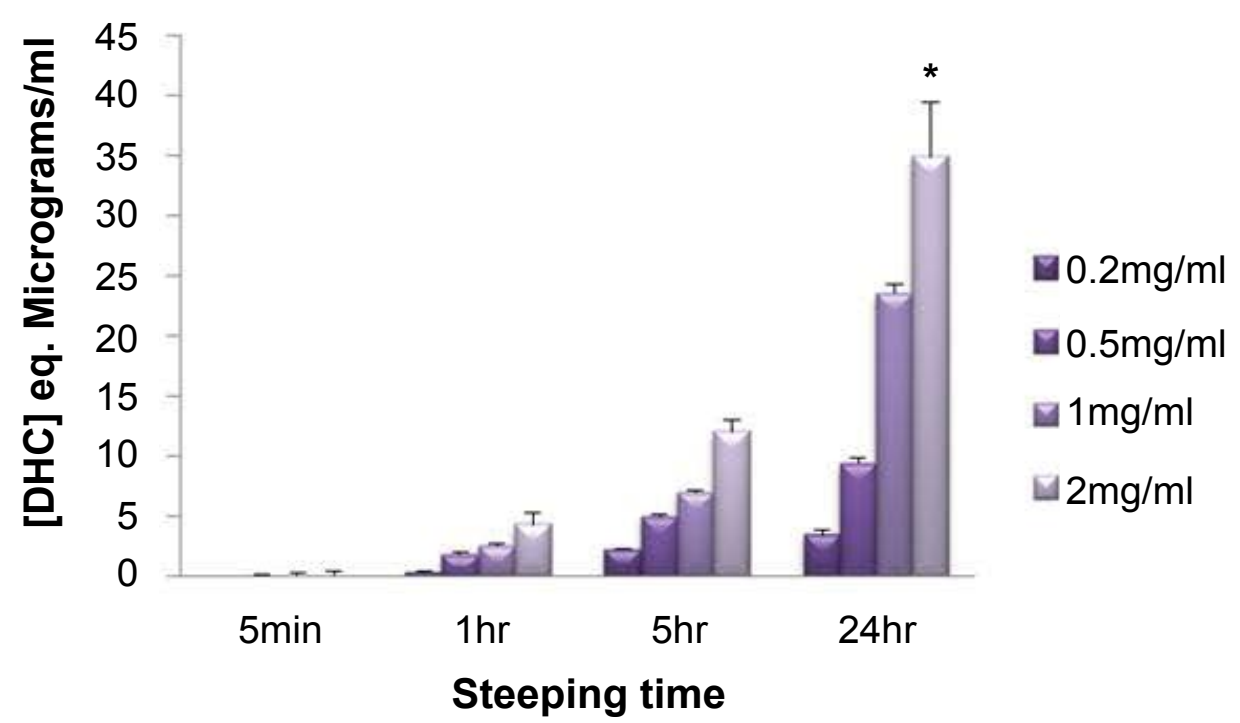

Figure 3: Polydopamine (PDA)-functionalisation of tissue culture plastic (TCP); a time and dose-response study. A series of dopamine hydrochloride $(\mathrm{DHC})$ solutions $(0.2-2.0 \mathrm{mg} / \mathrm{ml})$ were prepared using $10 \mathrm{mM}$ sodium bicarbonate $(\mathrm{pH}$ 8.3) as the buffer. Each of the different solutions were applied to TCP plates ( $1 \mathrm{ml} / \mathrm{well})$ and left for either 5 minutes, 1,5 or 24 hours. Following the incubation times TCP wells were aspirated and rinsed three times with distilled water prior to treatment with a bicinchoninic acid (BCA) assay reagent. After a 90-minute room temperature incubation aliquots (0.1 ml) of each sample were transferred to a 96-well plate and the absorbance at $540 \mathrm{~nm}$ taken against a standard series of DHC to extrapolate PDA at the TCP surfaces. All data (a representative from two independent experiments) are expressed as the mean equivalent of $\mathrm{DHC}$ (micrograms $/ \mathrm{ml}$ ) plus the standard deviation from 4 replicates. The findings depicted clearly indicate a time and dose-response effect for PDA-functionalisation of TCP with the $2 \mathrm{mg} / \mathrm{ml} \mathrm{DHC}$ solution for the longest exposure time yielding a TCP surface with the most PDA coverage (* $p<0.01$ versus all other DHC concentrations for a $24-$ hour treatment). 
Citation: Andrea A, Mansell JP (2018) A Facile and Sensitive Colorimetric Approach to Confirming the Presence of Polydopamine Thin Films on (Bio)Material Surfaces. Regen Med Ther 2(1):30-36
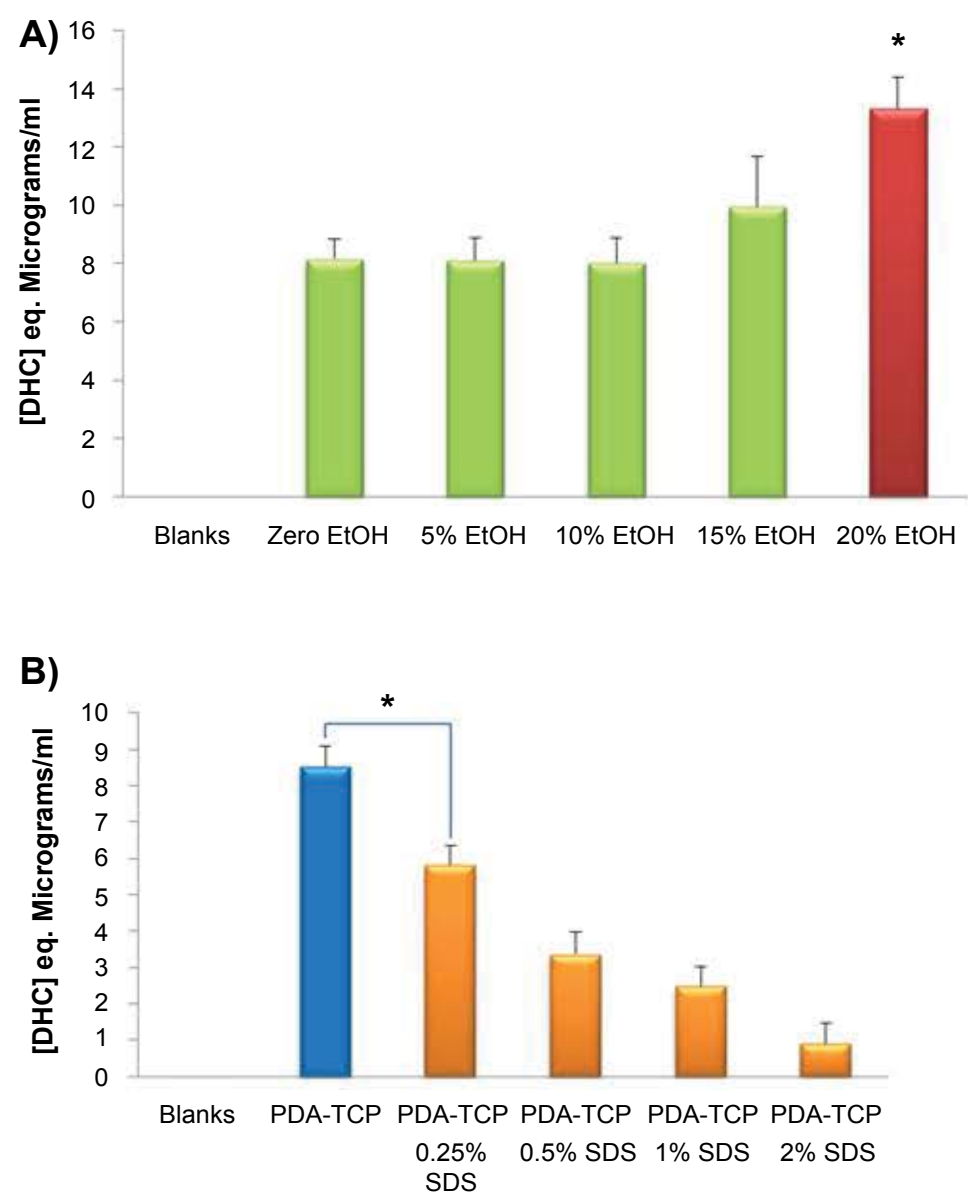

Figure 4: Ethanol (EtOH) enhances and sodium dodecyl sulphate (SDS) inhibits the extent of polydopamine (PDA)-functionalisation of tissue culture plastic (TCP).

A) A $2.5 \mathrm{mg} / \mathrm{ml}$ solution (4 ml) of dopamine hydrochloride (DHC) in $12.5 \mathrm{mM}$ sodium bicarbonate (pH 8.3) was mixed with either $1 \mathrm{ml}$ of distilled water, $1 \mathrm{ml} \mathrm{EtOH}$ or $1 \mathrm{ml}$ water: EtOH mixtures so that the effect of a range of different EtOH concentrations $(5-20 \% \mathrm{v} / \mathrm{v})$ on the ability of PDA to surface functionalise TCP could be examined. Each of the different solutions were applied to TCP plates $(0.9 \mathrm{ml} /$ well $)$ and left for 4 hours. Once treated TCP wells were aspirated and rinsed three times with distilled water prior to treatment with a bicinchoninic acid (BCA) assay reagent. After a 90-minute room temperature incubation aliquots $(0.1 \mathrm{ml})$ of each sample were transferred to a 96-well plate and the absorbance at $540 \mathrm{~nm}$ taken against a standard series of DHC to extrapolate PDA at the TCP surfaces. All data (a representative from two independent experiments) are expressed as the mean equivalent of $\mathrm{DHC}(\mathrm{micrograms} / \mathrm{ml})$ plus the standard deviation from 4 replicates. The findings depicted indicate that a final EtOH concentration of $20 \%(\mathrm{v} / \mathrm{v})$ supports the greatest extent of PDA coverage compared to all other groups ( $\left.{ }^{*} \mathrm{p}<0.01\right)$. B) A $2 \mathrm{mg} / \mathrm{ml}$ solution of DHC $(4.75 \mathrm{ml})$ was mixed with either $0.25 \mathrm{ml}$ of distilled water or $0.25 \mathrm{ml}$ of four different SDS solutions; $0.25 \%, 0.5 \%, 1.0 \%$ and $2.0 \%(\mathrm{w} / \mathrm{v})$. Each of the solutions were applied to TCP plates $(0.9 \mathrm{ml} / \mathrm{well})$, left for 4 hours and processed for PDA detection using the BCA assay reagent as detailed above. All data (a representative from two independent experiments) are expressed as the mean equivalent of $\mathrm{DHC}$ (micrograms $/ \mathrm{ml}$ ) plus the standard deviation from 4 replicates. The findings depicted indicate that SDS dose-dependently inhibits PDA formation at the TCP surface with a significantly reduced ( $\mathrm{p}<0.01$ ) PDA coating between the PDA-TCP control and the lowest SDS concentration used.

\section{PDA-functionalisation of TCP is enhanced using ethanol and inhibited by sodium dodecyl sulphate}

In a set of experiments, we examined the influence of ethanol on the ability of DHC to produce a PDA-functionalised TCP surface. A $2.5 \mathrm{mg} / \mathrm{ml}$ solution of DHC was prepared in $12.5 \mathrm{mM} \mathrm{NaHCO}_{3}$ and $4 \mathrm{ml}$ aliquots dispensed into $1 \mathrm{ml}$ volumes of either cell culture-grade water, ethanol, or water: ethanol blends to achieve final ethanol concentrations of $0,5,10,15 \& 20 \%(\mathrm{v} / \mathrm{v})$. Each of the different solutions $(0.9 \mathrm{ml})$ were dispensed into the wells of 24-well multiwell TC plates and left for 4 hours at room temperature prior to PDA detection. Ethanol at a final concentration of $20 \%(\mathrm{v} / \mathrm{v})$ yielded TCP surfaces with the greatest coverage of PDA compared to samples devoid of alcohol (Figure 4A). In marked contrast the inclusion of sodium dodecyl sulphate (SDS) inhibited the formation of PDA thin films in a dose-dependent manner (Figure $4 \mathrm{~B})$. Aliquots $(4.75 \mathrm{ml})$ of a $2 \mathrm{mg} / \mathrm{ml}$ solution of $\mathrm{DHC}$ in $10 \mathrm{mM} \mathrm{NaHCO}$ were dispensed into tubes containing $0.25 \mathrm{ml}$ of either water or aqueous SDS at starting concentrations between $0.25-2.0 \%(\mathrm{w} / \mathrm{v})$. Each of the different solutions $(0.9 \mathrm{ml})$ were dispensed into the 
Citation: Andrea A, Mansell JP (2018) A Facile and Sensitive Colorimetric Approach to Confirming the Presence of Polydopamine Thin Films on (Bio)Material Surfaces. Regen Med Ther 2(1):30-36

wells of 24-well multiwell TC plates and left for 4 hours at room temperature prior to PDA detection.

\section{Discussion}

Polydopamine is currently celebrating a "decade of discovery" [2]. Following the first report that PDA could be used to functionalise a plethora of materials [1] there has been a wealth of scientific reports detailing the fabrication of diverse, novel technologies. Developments in the PDA field include photonic materials [6], biosensors [7], drug delivery devices [8], antifouling platforms [9] and functional biomaterials for potential regenerative medicine applications [10].

Evidence for the successful binding of PDA thin films to different substrates typically involves a detailed physiochemical assessment to gauge, for example, coating thickness, uniformity and impact on surface wettability. The tools and approaches taken often necessitate the use of some rather sophisticated and technically sensitive instrumentation operated by highly skilled personnel. These include, but are not restricted to, atomic force microscopy [11], Raman spectroscopy [12], Fourier transform infra-red spectroscopy [13], contact angle measurements [14] and X-ray photoelectron spectroscopy analysis [15].

In developing our own PDA-functionalised Ti technology for bone regenerative applications we considered a pragmatic first (and facile) solution to confirming PDA formation at the metal surface. To this end we utilised a BCA-based assay procedure more commonly used for protein quantification. Herein we report that this inexpensive approach proved to be rather useful in corroborating the presence of PDA coatings at two different surfaces, TCP and Ti. Qualitatively we found evidence for a PDA-modification of TCP following a fifteen-minute exposure of a $2 \mathrm{mg} / \mathrm{ml}$ solution of DHC in sodium bicarbonate $(10 \mathrm{mM}, \mathrm{pH} 8.3)$. With increasing incubation time, the intensity of the violet complex generated using the BCA reagent increased (Figure 1). Next, we examined the influence of different buffers on PDA-functionalisation of TCP over a period of twenty hours (Figure 2). Mildly alkaline buffers consistently led to greater PDA coatings compared to neutral-buffered HEPES, findings in keeping with the current consensus that oxidation of dopamine, under alkaline conditions, is the limiting step to PDA formation. Indeed, when we reconstituted DHC in distilled water the resulting acidic solution was unable to support PDA formation upon TCP even after several days (data not shown). This was an important observation as it clearly indicated that the violet complex generated by the BCA reagent is not attributed to dopamine binding non-specifically to the material surface but rather the chemical process responsible for PDA generation and subsequent surface functionalisation by the polymer.
In addition, we explored the effect of varying the DHC concentration and exposure time to TCP on the development of a PDA coating. The findings presented (Figure 3) support a clear time and dose effect on the ability of PDA to modify the TCP surface. By introducing ethanol (Figure 4A) or SDS (Figure 4B) into solutions of DHC we were able to influence the extent to which PDA films formed at TCP surfaces. Altering the solvent properties, e.g., the dielectric constant could be explored to fine tune conditions to optimise and/or control PDA coatings. In our hands we find that ethanol, at a final concentration of $20 \%(\mathrm{v} / \mathrm{v})$, could significantly enhance PDA film formation. Our findings support the recent discovery that ethanol facilitates and enhances PDA formation by displacing the trapped air/vapour layer [16]. These findings will be of value in adjusting incubation times to speed up the PDA-functionalisation process. In light of the work of Ponzio and colleagues [17] we anticipated that the inclusion of SDS would inhibit PDA formation and we too found this to be the case, with the surfactant preventing PDA formation in a dose-dependent manner. Collectively the findings we present endorse the use of BCAbased assay reagents for the detection of PDA deposited thin films. The application of a BCA-based assay reagent affords a smooth and quick assessment of (bio)material functionalisation. Rather than default to a more rigorous physiochemical surface analysis from the outset the use of the BCA assay procedure provides greater flexibility towards substrate modification and optimisation; different buffer systems can be examined, incubation times and temperature settings, for example, can be effectively prosecuted in a high throughput format. Such an approach will make significant reductions in overall cost before taking the necessary steps towards a more comprehensive physiochemical assessment of the surface in overall PDA-based material design.

\section{Acknowledgements}

This work was supported by a short-term mission grant from the Med-Vet-Net Association, (ref 2017_ STM_9). The authors would also like to thank the University of The West of England, Bristol, for their research support and use of the laboratory facilities within the Centre for Research in Biosciences.

\section{Disclosures}

The authors have no conflicts of interest to declare.

\section{References}

1. Lee H, Dellatore SM, Miller WM, et al. (2007) Mussel-Inspired Surface Chemistry for Multifunctional Coatings. Science 318: 426-430.

2. Ryu JH, Messersmith PB, Lee H (2018) Polydopamine Surface Chemistry: A Decade of Discovery. ACS Appl Mater Interfaces 10: 7523-7540. 
Citation: Andrea A, Mansell JP (2018) A Facile and Sensitive Colorimetric Approach to Confirming the Presence of Polydopamine Thin Films on (Bio)Material Surfaces. Regen Med Ther 2(1):30-36

3. Schanze KS, Lee H, Messersmith PB (2018) Ten Years of Polydopamine: Current Status and Future Directions. ACS Appl Mater Interfaces 10: 7521-7522.

4. Alfieri ML, Micillo R, Panzella L, et al. (2017) Structural Basis of Polydopamine Film Formation: Probing 5,6-Dihydroxyindole-Based Eumelanin Type Units and the Porphyrin Issue. ACS Appl Mater Interfaces.

5. Liebscher J, Mrówczyński R, Scheidt HA, et al. (2013) Structure of polydopamine: a never-ending story? Langmuir 29: 10539-10548.

6. Razmjou A, Asadnia M, Ghabe O, et al. (2017) Preparation of Iridescent 2D Photonic Crystals by Using a Mussel-Inspired Spatial Patterning of ZIF-8 with Potential Applications in Optical Switch and Chemical Sensor. ACS Appl Mater Interfaces 9: 38076-38080.

7. Ji X, Yi B, Xu Y, et al. (2017) A novel fluorescent biosensor for Adenosine Triphosphate detection based on the polydopamine nanospheres integrating with enzymatic recycling amplification. Talanta 169: 8-12.

8. Liu Y, Sui Y, Liu C, et al. (2018) A physically crosslinked polydopamine/nanocellulose hydrogel as potential versatile vehicles for drug delivery and wound healing. Carbohydr Polym 188: 27-36.

9. Kim S, Gim T, Jeong Y, et al. (2018) Facile Construction of Robust Multilayered PEG Films on Polydopamine-Coated Solid Substrates for Marine Antifouling Applications. ACS Appl Mater Interfaces 10: 7626-7631.

10. Razavi M, Hu S, Thakor AS (2018) A collagen based cryogel bioscaffold coated with nanostructured polydopamine as a platform for mesenchymal stem cell therapy. J Biomed Mater Res A 106: 2213-2228.

11. Zhang Y, Wang W, Ma X, et al. (2016) Polydopamine assisted fabrication of titanium oxide nanoparticles modified column for proteins separation by capillary electrochromatography. Anal Biochem 512: 103-109.

12. Feng J, Fan H, Zha DA, et al. (2016) Characterizations of the Formation of Polydopamine-Coated alloysite Nanotubes in Various pH Environments. Langmuir 32: 1037710386.

13. Lim K, Chua RRY, Bow H, et al. (2015) Development of a catheter functionalized by a polydopamine peptide coating with antimicrobial and antibiofilm properties. Acta Biomaterialia 15: 127-138.

14. Liu CY, Huang CJ (2016) Functionalization of Polydopamine via the Aza-Michael Reaction for Antimicrobial Interfaces. Langmuir 32: 5019-5028.

15. He S, Zhou P, Wang L, et al. (2014) Antibiotic-decorated titanium with enhanced antibacterial activity through adhesive polydopamine for dental/bone implant. J R Soc Interface 11: 20140169

16. Zhang C, Gong L, Xiang L, et al. (2017) Deposition and Adhesion of Polydopamine on the Surfaces of Varying Wettability. ACS Appl Mater Interfaces 9: 30943-30950.

17. Ponzio F, Bertani P, Ball V (2014) Role of surfactants in the control of dopamine-eumelanin particle size and in the inhibition of film deposition at solid-liquid interfaces. J Colloid Interface Sci 431: 176-179. 